\title{
NUMERICAL MODELING OF THE INITIATION OF CORONAL MASS EJECTIONS IN ACTIVE REGION NOAA 9415
}

\author{
F. P. Zuccarello ${ }^{1,2,3}$, Z. Meliani $^{4}$, and S. Poedts ${ }^{1}$ \\ ${ }^{1}$ Centre for Mathematical Plasma-Astrophysics, KU Leuven, Celestijnenlaan 200B, B-3001 Leuven, Belgium; \\ Francesco.Zuccarello@wis.kuleuven.be, Stefaan.Poedts@wis.kuleuven.be \\ ${ }^{2}$ Dipartimento di Fisica e Astronomia, Universitá di Catania, via S. Sofia 78, I-95123 Catania, Italy \\ ${ }^{3}$ INAF-Osservatorio Astrofisico di Catania, via S. Sofia 78, I-95123 Catania, Italy \\ ${ }^{4}$ Observatoire de Paris, LUTh, F-92190 Meudon, France; zakaria.meliani@ obspm.fr \\ Received 2012 April 4; accepted 2012 August 11; published 2012 October 8
}

\begin{abstract}
Coronal mass ejections (CMEs) and solar flares are the main drivers of weather in space. Understanding how these events occur and what conditions might lead to eruptive events is of crucial importance for up to date and reliable space weather forecasting. The aim of this paper is to present a numerical magnetohydrodynamic (MHD) data-inspired model suitable for the simulation of the CME initiation and their early evolution. Starting from a potential magnetic field extrapolation of the active region (AR) NOAA 9415, we solve the full set of ideal MHD equations in a non-zero plasma- $\beta$ environment. As a consequence of the applied twisting motions, a force-freemagnetic field configuration is obtained, which has the same chirality as the investigated AR. We investigate the response of the solar corona when photospheric motions resembling the ones observed for AR 9415 are applied at the inner boundary. As a response to the converging shearing motions, a flux rope is formed that quickly propagates outward, carrying away the plasma confined inside the flux rope against the gravitational attraction by the Sun. Moreover, a compressed leading edge propagating at a speed of about $550 \mathrm{~km} \mathrm{~s}^{-1}$ and preceding the CME is formed. The presented simulation shows that both the initial magnetic field configuration and the plasma-magnetic-field interaction are relevant for a more comprehensive understanding of the CME initiation and early evolution phenomenon.
\end{abstract}

Key words: magnetohydrodynamics (MHD) - methods: numerical - Sun: corona - Sun: coronal mass ejections (CMEs)

Online-only material: color figures

\section{INTRODUCTION}

The solar corona is a magnetically dominated environment where "open" field magnetic regions, such as coronal holes, and closed field regions, such as active regions (ARs), coexist. However, it is not uncommon that closed magnetic field regions open up, eventually resulting in eruptive events such as coronal mass ejections (CMEs) and flares. Understanding how these events occur and which conditions may lead to eruptive events is crucial for accurate and reliable space weather forecasting. Observations show that quite often, CMEs and flares occur in the presence of highly sheared magnetic fields. Many examples of this can be found in the literature. For example, Asai et al. (2009) studied AR 10798, which was the source of multiple M-class flares and CMEs in late 2005 August. This AR evolved from a dipole-like configuration to a more sheared configuration before erupting. Cristiani et al. (2007) investigated AR 9715, which produced an M-class flare on 2001 November 28. By combining EUV, $\mathrm{H} \alpha$, magnetogram images, and linear forcefree field extrapolations, these authors concluded that the AR had a significant shear at the moment of the eruption, and the higher shear was observed closer to the filament. Sunspot rotations are also commonly observed, as reported, for example, by Zhang et al. (2007). The major solar event of 2006 December 13 took place after AR 10930 had rotated by about $200^{\circ}$ around its center.

Photospheric convergence motions toward the polarity inversion line, resulting in flux cancellation events, are also often observed to precede flares and CMEs. Sterling et al. (2010), investigating a filament eruption which occurred on 2007 May 20, observed persistent convergence motions of positive magnetic flux concentrations toward the polarity inversion line, which eventually resulted in episodes of flux cancellation. The authors concluded that the tether-cutting reconnection driven by these convergence motions may have led to the destabilization of the filament. A major flux cancellation event, canceling about one-third of the AR's magnetic flux, which resulted in the formation of a sigmoid and in its eventual eruption, was reported by Green et al. (2011). These authors showed that flux cancellation occurred when the negative magnetic flux of AR 10977 was transported toward the main polarity inversion line. Similar dynamics may have occurred for the 2008 April 26 event (Cheng et al. 2010). Recently, Su et al. (2011) investigated the flare/CME event that occurred in AR 11060 on 2010 April 8, using both space-born observations and nonlinear forcefree (NLFF) field models. They concluded that the event may have been initiated by a catastrophic loss of equilibrium caused by the increase in the axial flux in the flux rope, which was driven by flux cancellation events. These are only a few examples of the observations available in the literature. They point out the relevance of converging photospheric motions toward the polarity inversion line as precursors of flare/CME activity. Statistically, there is a positive correlation between flux cancellation and flare/CME productivity of ARs. However, Welsch et al. (2011) investigated three major ARs and found three exceptions to this statistically significant correlation. Therefore, further investigations from both an observational and a theoretical point of view are necessary in order to discern in which cases flux cancellation results in violent events and in which cases it does not. 
From a theoretical point of view, van Ballegooijen \& Martens (1989) proposed flux cancellation, driven by photospheric convergence motions toward the polarity inversion line of a sheared arcade configuration, as a possible mechanism to transfer the arcade field into the helical field. The authors showed that, starting from an initial force-free-magnetic field, a new magnetic field configuration in which the axis of the helical field moves to larger heights can be obtained by converging photospheric shearing motions. In an axisymmetric configuration, Forbes \& Priest (1995) considered the effect of convergence motions toward the polarity inversion line when a flux rope model (Forbes \& Isenberg 1991) is considered. When the photospheric magnetic sources approach one another, the magnetic energy stored in the flux rope increases until a critical point is reached and the system undergoes a loss of equilibrium resulting in an eruption.

Convergence shearing motions and their relationship with flux cancellation have been widely studied in fully threedimensional magnetic field configurations. For example, Amari et al. (2003a) considered the potential magnetic field generated by two symmetrically located magnetic sources. In order to obtain an NLFF configuration, at the lower boundary, the authors applied vortex motions along the isocontours of the normal component of the magnetic field and let the system relax to the desired force-free equilibrium. This equilibrium consists of highly sheared field lines close to the polarity inversion line and twisted field lines away from it. In a second phase, both polarities have been subjected to photospheric convergence motions toward the polarity inversion line. As a result, magnetic reconnection sets in between the highly sheared field lines resulting in the formation of a twisted flux rope. They found that the rope is never in equilibrium and experiences a full eruption. In a recent paper, Amari et al. (2011) reconsidered the same initial magnetic field configuration, but applied radially diverging flows centered around the two magnetic polarities instead of applying convergence motions over all of the magnetic sources. In the region between the two magnetic polarity centers, these flows converge toward the polarity inversion line. As a result, an equilibrium including a flux rope is found. However, after some time, the system suffers a full disruption. This simulation shows that in order to drive an eruption, it is not necessary to advect the full AR toward the polarity inversion line.

As far as modeling is concerned, the effect of flux cancellation can also be modeled by applying a prescribed time-dependent boundary condition on the normal component of the magnetic field. For example, Amari et al. (2000) showed that a sheared arcade can undergo an eruption when a new flux of opposite sign emerges from below the photosphere. A similar disruption can also be obtained when turbulent diffusion is considered (Amari et al. 2003b). The effect of turbulence is to disperse the magnetic flux of the AR, which is then canceled, by smallscale mixing. In a recent simulation, Aulanier et al. (2010) considered the evolution of an asymmetric, bipolar AR subjected to both twisting motions and enhanced photospheric diffusion. The twisting motions allow the formation of highly sheared field lines along the polarity inversion line, while the effect of the diffusive term is to diffuse the three components of the magnetic field resulting in a horizontal expansion of both polarities. This process, near the polarity inversion line, leads to the annihilation of the vertical component of the magnetic field and can result in a formation of a flux rope. In their simulation, the eruption is driven by the torus instability and not by the tether-cutting reconnection. The equivalence between the different flux cancellation approaches has been shown by Amari et al. (2010). The authors proved that the imposed flux disappearance at the lower boundary can be interpreted in terms of convergence motions toward the polarity inversion line. The authors also found that only a modest decrease in flux is sufficient to drive an eruption.

The upward gradient of the magnetic field in the solar corona is strictly related to the variation of the plasma- $\beta$ with height. The plasma- $\beta$ measures the relative strength of the gas pressure with respect to the magnetic pressure. It is often assumed that $\beta \ll 1$ in the solar corona, and the dynamic is therefore dominated by the magnetic field. Starting from this assumption, most of the numerical simulations discussed above have been performed assuming $\beta=0$, namely, neglecting the gas pressure and the plasma response. However, as shown by Gary (2001), in the low corona, i.e., at a height of about $1.25 R_{\odot}$, the plasma- $\beta$ can be of the order of one. Quite extended regions, where $\beta>1$ at an altitude of about $1.5 R_{\odot}$, are also found in global magnetohydrodynamic (MHD) simulations when complex physical processes, such as thermal conduction along the field lines, radiative losses, Alfvén wave pressure, and a parametric heating term (Lionello et al. 2009), are considered (Z. Mikić et al. 2012, private communication). From a theoretical point of view, the importance of finite- $\beta$ plasmas in the solar environment has been discussed in Fuentes-Fernández et al. (2010). Investigating the relaxation of a perturbed two-dimensional magnetohydrostatic state, these authors found that the effect of finite plasma- $\beta$ leads to significant differences from the zero- $\beta$ case. Furthermore, when zero- $\beta$ models are considered, during reconnection events the magnetic energy decrease cannot be converted into heat or into particle acceleration (Aulanier et al. 2012). However, during a solar eruption, both processes are relevant. Therefore, in order to fully understand the initiation of CMEs and their early evolution in a more realistic solar corona, non-zero- $\beta$ simulations are necessary.

The aim of this paper is to extend the results achieved in the latter class of models by introducing a more realistic solar atmosphere that includes the presence of plasma, eventually enabling us to investigate the shock formation that is often observed during the CME liftoff (Mancuso 2011; Gopalswamy \& Yashiro 2011). As in Amari et al. (2003a) and Aulanier et al. (2010), we consider a potential magnetic field in a Cartesian domain that represents a portion of the solar corona between $1.1 R_{\odot}$ and $1.9 R_{\odot}$. In our work, the potential field is the result of an extrapolated magnetic field. In particular, we consider the magnetic field of the AR NOAA 9415. This approach is the natural consequence of several research efforts. In fact, Amari et al. (2003a) considered a symmetric dipole, while Aulanier et al. (2010) considered an asymmetric dipole, and here we consider a fully asymmetric magnetic field configuration that is directly related to the observations. With respect to Amari et al. (2003a) and Aulanier et al. (2010), in our work, the magnetic field is embedded in a finite plasma- $\beta$ environment in hydrostatic equilibrium. In a similar fashion as Amari et al. (1996, 2003a, 2011), we apply photospheric twisting motions in order to create an NLFF state that resembles the magnetic structure of AR 9415. Starting from the results of Amari et al. (2011), and based on the observations of the photospheric magnetic field evolution of the AR 9415, in this work we investigate the effect of a new class of convergence motions in which only one magnetic polarity is advected toward the polarity inversion line. With the present work, we aim (1) to investigate whether or not this new class of convergence motions can trigger 


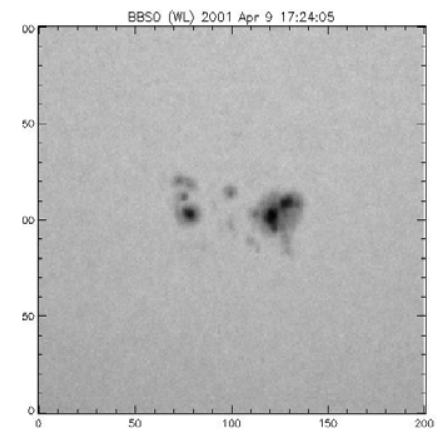

(a)

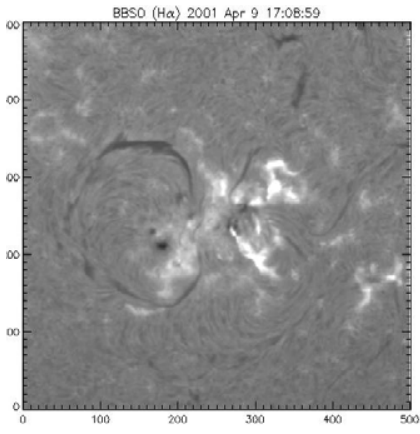

(b)

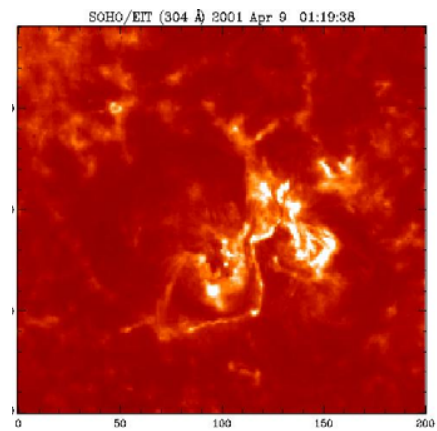

(c)

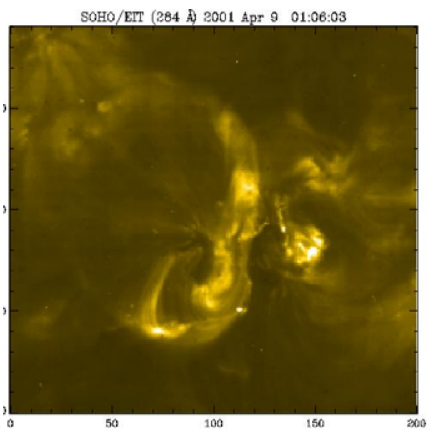

(d)

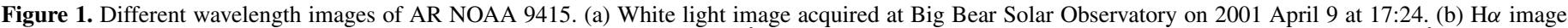

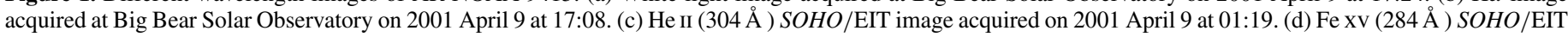
image acquired on 2001 April 9 at 01:06. North is at the top and west to the right.

(A color version of this figure is available in the online journal.)

an eruption, (2) what is the effect of the finite- $\beta$ in the evolution of the system, and (3) to present a new data-inspired approach to the modeling of the CME initiation and early evolution.

The structure of the paper is as follows. In Section 2, we briefly describe the morphology of the AR and the CMEs related to it. The simulation setup is described in Section 3 together with the applied initial and boundary conditions. The results of the simulations are presented in Section 4 and discussed in Section 5. A summary concludes our paper.

\section{ACTIVE REGION MORPHOLOGY}

On 2001 April 3, AR NOAA 9415 appeared on the east limb of the Sun at a latitude of about $22^{\circ}$ south, crossed the central meridian on April 9 at 12:48 UT, and was visible on the solar disk until April 14. During its transit, AR 9415 was the source of several CMEs and class B, C, M, and X flares, with the strongest ones occurring on April 6 at 19:10 UT (X 5.6) and April 10 at 05:06 UT (X 2.3). Figure 1 shows white light, $\mathrm{H} \alpha$, He II ( $\lambda 304)$, and Fe XV ( $\lambda 284)$ images of AR 9415 taken on 2001 April 9, when the AR crossed the central meridian. The AR presents a quite complex configuration: the two sunspots that share the same penumbra are well separated from the following sunspot. Several pores are seen between the two preceding sunspots and surrounding the following one. Figure 2 (top panel) shows the magnetic configuration of the AR on April 9 at 00:00 UT. The preceding sunspots are characterized by a magnetic field of opposite polarities, forming a $\delta$-spot. The following sunspot is characterized by a positive magnetic flux distribution and is surrounded by a larger ring of the same magnetic polarity. Many filaments are present in the AR (Figure 1(b)), and the largest ones are located northeast and southeast of the following polarity. In particular, the southeast filament, which is located above the neutral line between the $\delta$-spot and the positive flux ring, starts to rise on April 10 at around 04:00 UT and undergoes a full eruption at 05:06 UT, resulting in a halo CME. Figure 1(c) shows the EUV filament channel associated with this filament, while Figure 1(d) shows a J-shaped loop system, rather than a fully developed sigmoid, connecting the $\delta$-spot to the following polarity.

Figure 2 shows a sequence of co-aligned MDI magnetograms, reporting the magnetic configuration of NOAA 9415, which were taken between 2001 April 9-11. A clear east-west shearing motion of the following positive polarity, specifically the central part of it, is observed, while the negative polarity mainly maintains both its position and shape. A northeast motion of the positive polarity of the $\delta$-spot that likely results in a further injection of magnetic shear in the configuration is also evident.

In the following sections of this paper, we investigate, by means of numerical MHD simulations, the vulnerability of a magnetic field configuration, resembling the one of AR 9415 when the system is driven by convergence shearing motions, resembling the ones described above.

\section{SIMULATION SETUP}

In order to model the evolution of AR 9415 in a Cartesian domain, we solve the full set of ideal MHD equations:

$$
\begin{aligned}
\frac{\partial \rho}{\partial t}+\nabla \cdot(\rho \mathbf{v}) & =0 \\
\frac{\partial \rho \mathbf{v}}{\partial t}+\nabla \cdot(\rho \mathbf{v v}-\mathbf{B B})+\nabla\left(p+\frac{\mathbf{B}^{2}}{2}\right) & =-\rho \nabla \Phi_{\mathrm{G}}, \\
\frac{\partial e}{\partial t}+\nabla \cdot\left[\left(e+p+\frac{B^{2}}{2}\right) \mathbf{v}-\mathbf{B}(\mathbf{v} \cdot \mathbf{B})\right] & =-\rho \mathbf{v} \cdot \nabla \Phi_{\mathrm{G}}, \\
\frac{\partial \mathbf{B}}{\partial t}+\nabla \cdot(\mathbf{v B}-\mathbf{B v}) & =0
\end{aligned}
$$

where

$$
e=\frac{p}{\gamma-1}+\rho \frac{v^{2}}{2}+\frac{B^{2}}{2}
$$

is the total energy density, $\rho$ is the density, $\mathbf{v}$ is the velocity, $\mathbf{B}$ is the magnetic field, $p$ is the thermal pressure, and $\Phi_{G}$ is the gravity potential created by the Sun.

The three-dimensional Cartesian domain of $552^{3} \mathrm{Mm}$ is resolved using $256^{3}$ grid points, i.e., implementing a spatial resolution similar to the MDI magnetograms, and covers the solar corona from about $1.1 R_{\odot}$ to $1.9 R_{\odot}$.

The simulations are performed with the Message Passing Interface-Adaptive Mesh Refinement Versatile Advection Code (MPI-AMRVAC; Keppens et al. 2012). MPI-AMRVAC is a finite volume, cell-centered code suitable for solving any system of hyperbolic partial differential equations, such as the classical MHD equations (Equations (1)). Several temporal discretization and shock capturing schemes are implemented in the code. For the simulations discussed in the present paper, a Harten, Lax, and van Leer flux formulation (HLL; Harten et al. 1983) with a piecewise parabolic method (Colella \& Woodward 1984) limiter was used. Even though the HLL solver computes the fluxes only 

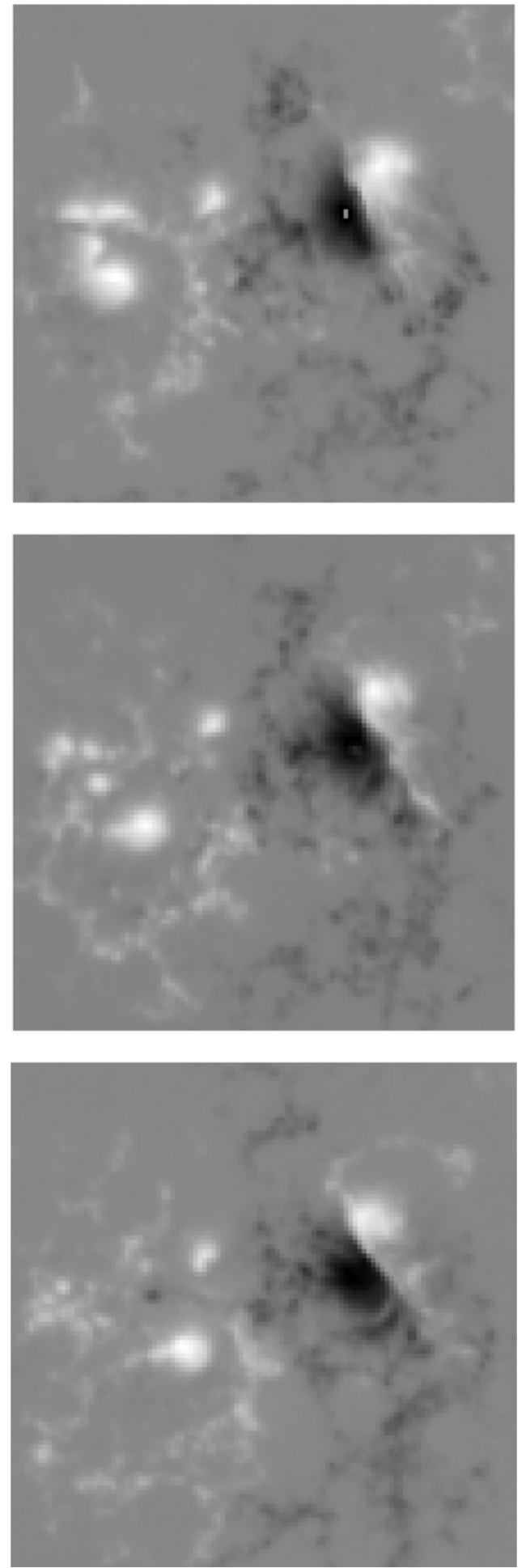

Figure 2. Sequence of co-aligned MDI magnetograms of AR NOAA 9415 taken on 2001 April 9 at 00:00 UT (top), April 10 at 04:48 UT (middle), and April 11 at 08:00 UT (bottom). North is at the top and west to the right.

using selected waves of the Riemann fan (Keppens et al. 2012), it is a robust scheme that allow us to decrease the numerical noises that may affect the regions with low plasma- $\beta$. The hyperbolic divergence cleaning (Dedner et al. 2002) algorithm is used to ensure the divergence free property of the magnetic field and the code has been modified in such a way that only the deviations from the current-free potential field $\mathbf{B}_{0}$ are advanced in time (Tanaka 1994; Powell et al. 1999). Finally, the boundary conditions are applied in $n$-layers (where $n$ depends on the order of the scheme) of ghost cells that surround the computational domain. In this way, the boundary conditions are fully specified in the subroutines updating the ghost cells, and the boundary conditions become independent of the choice of the numerical algorithms.

\subsection{Initial Condition}

As an initial condition for the magnetic field, we performed a potential field extrapolation of the AR following an approach similar to Otto et al. (2007). We considered a Fourier decomposition of the MDI magnetogram using the first 16 modes in order to resolve the large-scale magnetic structures of the AR. Afterward, we reconstructed the potential magnetic field using a set of base functions that satisfies the line mirroring symmetry condition with respect to the central line for each of the four lateral boundary planes. In fact, as shown by Otto et al. (2007), this type of symmetry condition for the magnetic field satisfies the symmetry properties of the MHD equations without violating the force-free condition at the boundaries. With this assumption, the three components of the magnetic field are

$$
\begin{aligned}
& B_{x}(x, y, z)=\sum_{m+n=e v e n} \frac{\exp \left(-\lambda_{m n} z\right)}{\kappa^{2}+\lambda_{m n}^{2}} \\
& \quad \times\left[c_{m n}^{(1)}\left(\gamma_{n} \kappa \sin \alpha_{m} x \cos \gamma_{n} y-\alpha_{m} \lambda_{m n} \cos \alpha_{m} x \sin \gamma_{n} y\right)\right. \\
& \left.\quad+c_{m n}^{(4)}\left(\alpha_{m} \lambda_{m n} \sin \alpha_{m} x \cos \gamma_{n} y-\gamma_{n} \kappa \cos \alpha_{m} x \sin \gamma_{n} y\right)\right]
\end{aligned}
$$

$$
\begin{aligned}
& B_{y}(x, y, z)=\sum_{m+n=e v e n} \frac{\exp \left(-\lambda_{m n} z\right)}{\kappa^{2}+\lambda_{m n}^{2}} \\
& \quad \times\left[c_{m n}^{(1)}\left(-\gamma_{n} \lambda_{m n} \sin \alpha_{m} x \cos \gamma_{n} y-\alpha_{m} \kappa \cos \alpha_{m} x \sin \gamma_{n} y\right)\right. \\
& \left.\quad+c_{m n}^{(4)}\left(\alpha_{m} \kappa \sin \alpha_{m} x \cos \gamma_{n} y+\gamma_{n} \lambda_{m n} \cos \alpha_{m} x \sin \gamma_{n} y\right)\right]
\end{aligned}
$$

$$
\begin{aligned}
B_{z}(x, y, z)= & \sum_{m+n=e v e n} \exp \left(-\lambda_{m n} z\right)\left[c_{m n}^{(1)} \sin \alpha_{m} x \sin \gamma_{n} y\right. \\
& \left.+c_{m n}^{(4)} \cos \alpha_{m} x \cos \gamma_{n} y\right],
\end{aligned}
$$

where $c_{m n}^{(1)}$ and $c_{m n}^{(2)}$ are the Fourier coefficients derived from the magnetogram, $\alpha_{m}=\pi m / L_{x}, \gamma_{n}=\pi n / L_{y}$, and $\lambda_{m n}^{2}=$ $\alpha_{m}^{2}+\gamma_{n}^{2}-\kappa^{2}$, where $\kappa$ is the force-free parameter. In order to consistently use the $\mathbf{B}_{0}$-splitting approach, a potential field extrapolation has been considered, yielding $\kappa=0$.

The extrapolated magnetic field is superimposed to an isothermal, $T_{0}=1.5 \times 10^{6} \mathrm{~K}$, hydrostatic equilibrium. The initial density profile is

$$
\rho(z)=\rho(z=0) \exp \left[\frac{G M_{\odot}}{v_{0}^{2}}\left(\frac{1}{z+R_{\odot}}-\frac{1}{R_{\odot}}\right)\right],
$$

where $z$ is the altitude from the photosphere, $G$ is the gravitational constant, $M_{\odot}$ and $R_{\odot}$ are the solar mass and radius, respectively, $v_{0}=157 \mathrm{~km} \mathrm{~s}^{-1}$ is the isothermal sound speed, and $\rho(z=0)=1 \times 10^{-16} \mathrm{~g} \mathrm{~cm}^{-3}$, i.e., the average low corona density. With these assumptions, the initial pressure profile is

$$
p(z)=p(z=0) \exp \left[\frac{G M_{\odot}}{v_{0}^{2}}\left(\frac{1}{z+R_{\odot}}-\frac{1}{R_{\odot}}\right)\right],
$$




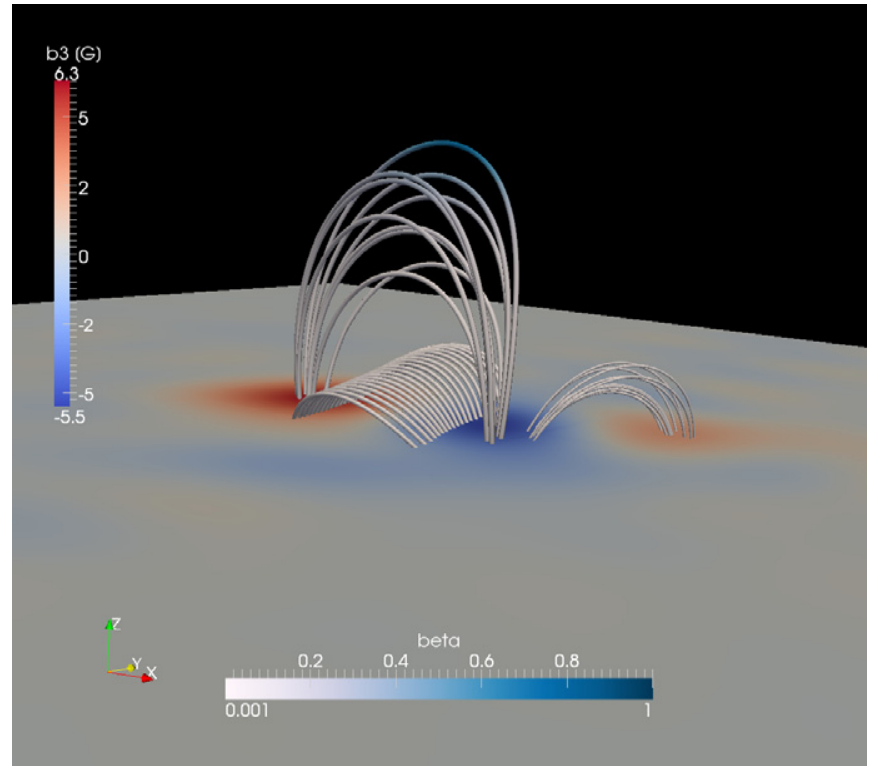

(a)

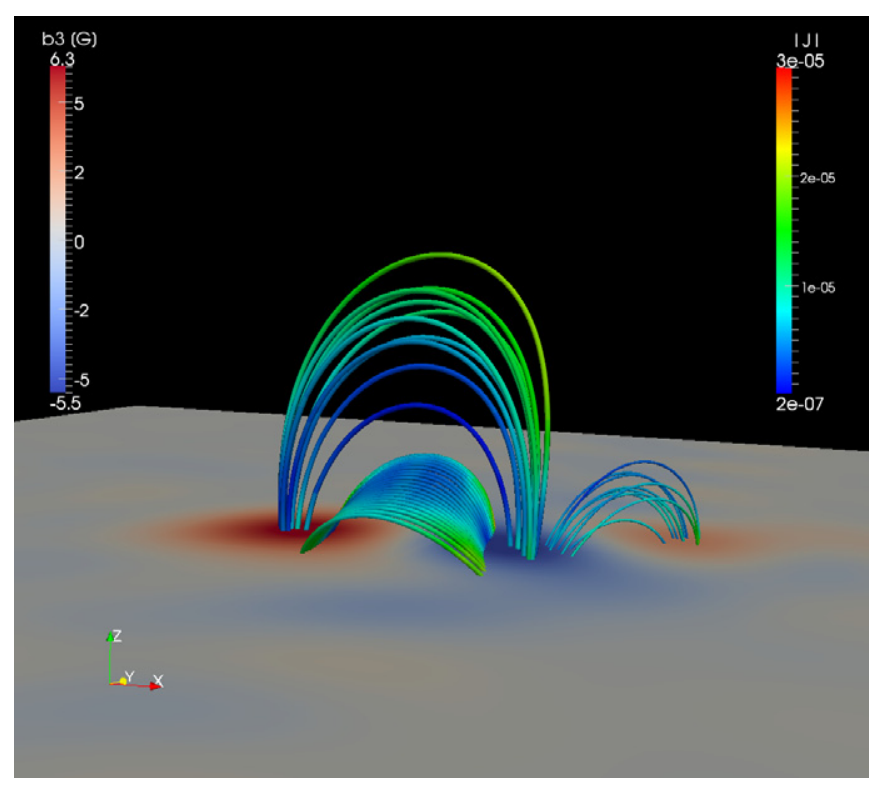

(b)

Figure 3. Initial condition for the simulation, when the magnetohydrostatic equilibrium has been reached. Distribution of the vertical component of the magnetic field and selected field lines color coded with the (a) plasma- $\beta$ and with the (b) current density.

(A color version of this figure is available in the online journal.)

where $p(z=0)=v_{0}^{2} \rho(z=0)$. The velocity field is initially imposed to zero $(\mathbf{v}=\mathbf{0})$.

Keeping the vertical component of the magnetic field, the density and the pressure fixed at the lower boundary, and also prescribing the pressure and the density at the upper boundary, we let the system relax toward numerical equilibrium. Figure 3 shows such a relaxed magnetohydrostatic equilibrium. In particular, Figure 3(a) shows a few selected field lines of the extrapolated magnetic field color coded with the plasma$\beta$, superimposed to the vertical component of the magnetic field. Similar to the MDI magnetogram, the field distribution is characterized by three flux concentrations. As a consequence of the limited number of modes that we use, the small-scale structures are not reproduced in the extrapolated magnetogram.
However, as already mentioned, we are interested in the coronal evolution of the magnetic field where only the large-scale structures are relevant. Due to numerical limitations, the magnetic field component is further reduced in order to maintain a reasonable time step and the magnetic field is scaled to have an intensity of about 5-7 G at the inner boundary. This is in agreement with magnetic field measurements above ARs and at a height of about $70 \mathrm{Mm}$ above the photosphere (Lin et al. 2004). The field lines shown in Figure 3(a) extend up to a height of about $1.25 R_{\odot}$ above the photosphere. As shown in the figure, the plasma- $\beta<1$ upto $1.25 R_{\odot}$, however, due to the scaling of the magnetic field discussed above, the plasma- $\beta>1$ higher up in the numerical box, in agreement with the conclusions of Gary (2001) and with other finite- $\beta$ MHD simulations (Z. Mikić et al. 2012, private communication). Finally, even though the velocity field is initialized with the condition $\mathbf{v}=\mathbf{0}$, when the system evolves, it relaxes to a steady state characterized by flows of about $10^{-2} \mathrm{~km} \mathrm{~s}^{-1}$, i.e., about four orders of magnitude smaller than the sound speed. This is a consequence of the way in which the gravity term and the pressure gradient are introduced in the MHD equations. The gravity is introduced as a source term and therefore it is calculated in the cell centers, while the pressure gradient is calculated at the cell surfaces. This mismatch results in a inexact balance between the gravity force and the pressure gradient that is responsible for the flows. Onedimensional hydrodynamic test simulations were performed in order to study the effect of the grid resolution on the amplitude of the flow: doubling the grid resolution reduces the flows by about one order of magnitude.

\subsection{Boundary Conditions}

In order to simulate the eruption, in the present paper we follow a four-step approach. First, a relaxed hydrostatic equilibrium is obtained. Second, we superimpose on it the extrapolated potential magnetic field and let the system relax toward a magnetohydrostatic equilibrium. Third, photospheric twisting motions are applied at the lower boundary in order to build up electric currents. Finally, convergence motions, resembling the one observed for the $\mathrm{AR}$, are applied in order to drive the eruption. During these four phases, different boundary conditions are imposed to the lower (photospheric or, rather, low coronal) boundary of the simulation box.

To obtain the hydrostatic equilibrium, at the lower boundary $z=0$, i.e., at $1.1 R_{\odot}$, the density and the pressure are fixed to their low corona values, and for the velocity field reflecting boundary conditions are selected, and, as a consequence, $\mathbf{v}=\mathbf{0}$ at the cell faces. A similar Dirichlet condition is imposed at the upper boundary as well. During this phase I, the dynamic mainly develops along the vertical component and does not influence the tangential component of the velocity, i.e., $v_{x}=v_{y}=0$ in the whole domain.

On the relaxed hydrostatic equilibrium, we superimpose the extrapolated magnetic field and let the system relax. This is phase II of the simulation. At the lower boundary, the density and the pressure are fixed, as in the previous phase. For the tangential component of the velocity, the condition $\mathbf{v}_{/ /}=\mathbf{0}$ at the cell faces is imposed, and since $v_{z}$ is several orders of magnitude smaller than the sound speed, this is similar to impose $\mathbf{E}_{/ /}=\mathbf{0}$, which is a well-posed boundary condition when relaxation runs are performed (Amari et al. 2003a, 2010). For the vertical component of the velocity $v_{z}$ and for the tangential component of the magnetic field $\mathbf{B}_{/ /}$, the Neumann-type zero derivative condition is imposed. Finally, the vertical component of the 


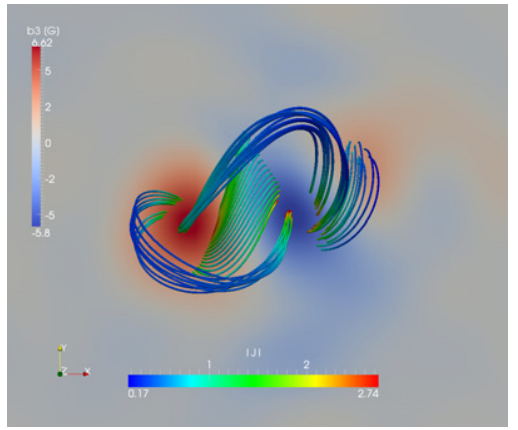

(a)

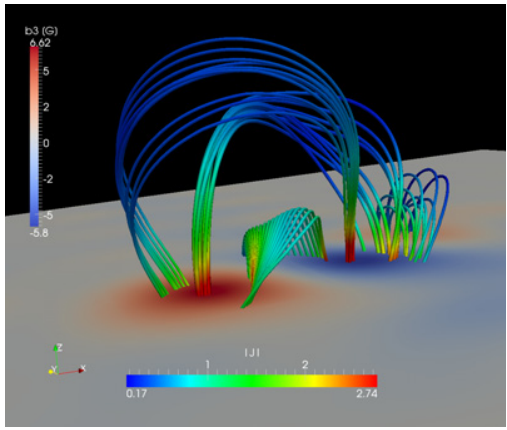

(b)

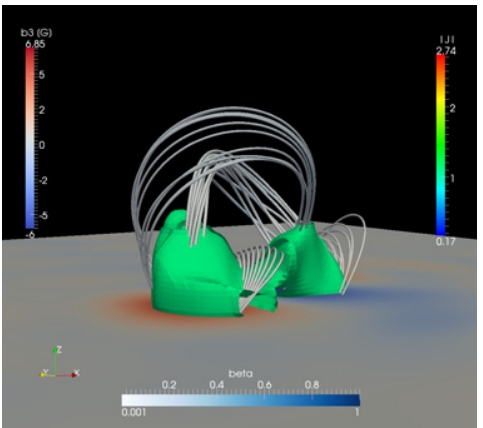

(c)

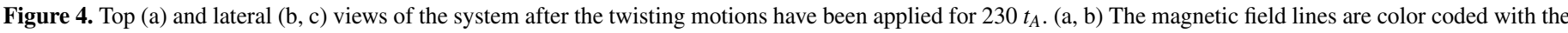

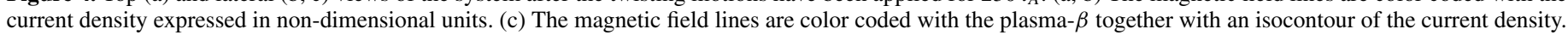
(A color version of this figure is available in the online journal.)

magnetic field $B_{z}$ is fixed through the solenoidal condition. The obtained magnetohydrostatic equilibrium was discussed in the previous section.

Phases I and II are needed to obtain the initial condition for the simulation. During these phases, no currents are introduced into the system except the negligible contribution due to the plasma flow. Figure 3(b) shows the current distribution after the relaxed state is obtained. As evident from the figure and as expected from the potential nature of the initial magnetic field, only minor currents are present in the system as a consequence of the interaction between the plasma and the magnetic field. However, these currents never exceeded $3 \times 10^{-5}$ (the current density will be expressed in non-dimensional units), with the highest value being at the lower boundary.

In phase III, we aim to build up currents in the system in order to have enough free-magnetic energy to drive the eruption. Following an approach similar to Amari et al. (1996, 2003a, 2010, 2011), Török \& Kliem (2003), and Aulanier et al. (2005), we apply localized vortex motions around the three main polarities of the AR. The applied twisting profile is

$$
\begin{aligned}
v_{x}(x, y, t)= & -2 v_{0} v_{A} \exp \left(\frac{B_{z}^{2}-B_{\max }^{2}}{\delta B^{2}}\right) \\
& \times\left(1+\frac{B_{z}^{2}-B_{\max }^{2}}{\delta B^{2}}\right) B_{z} \frac{\partial B_{z}}{\partial y}, \\
v_{y}(x, y, t)= & +2 v_{0} v_{A} \exp \left(\frac{B_{z}^{2}-B_{\max }^{2}}{\delta B^{2}}\right) \\
& \times\left(1+\frac{B_{z}^{2}-B_{\max }^{2}}{\delta B^{2}}\right) B_{z} \frac{\partial B_{z}}{\partial x},
\end{aligned}
$$

where $v_{0}=4 \times 10^{-4}$ is chosen in such a way that the maximum speed never exceeds $5 \%$ of the local Alfvén speed, $B_{\max }$ is the maximum of the normal component of the magnetic field at the inner boundary, and $\delta B=5 B_{\max }$ and $v_{A}$ is the Alfvén speed. These boundary motions are gradually switched on using a linear ramp up function. This velocity profile was already discussed by other authors and preserves the vertical component of the magnetic field. In fact, it is applied along the isocontours of $B_{z}$. In order to mimic the higher density of the photosphere, the boundary condition on the normal component of the velocity is designed in such a way that inflows into the Sun are limited to be of the order of $10 \%$ of the Alfvén speed. The boundary conditions for the other MHD variables are the same as in phase II.

In order to mimic the observed converging motions, in phase IV we impose photospheric shearing motions centered around the east-most positive polarity. In particular, within a radius $r(x, y)=\left(\left(x-x_{0}\right)^{2}+\left(y-y_{0}\right)^{2}\right)^{1 / 2}<r_{0}$, with $x_{0}$ and $y_{0}$ being the coordinates of the center of the positive polarity and $r_{0}$ its average radius, the following shearing profile is applied:

$$
v_{x}=v_{\max } v_{A} \cos \left(\frac{\pi r(x, y)}{2 r_{0}}\right),
$$

where $v_{\max }=0.05$, i.e., convergence motions are never larger than $5 \%$ of the local Alfvén speed and $v_{y}$ is fixed to zero. As in phase III, the photospheric motions are gradually switched on using a linear ramp function and the full photospheric speed is reached after $110 t_{A}$. Note that even though these values are still greater than the typical photospheric motions, they are significantly slower than the Alfvén speed. Therefore, a quasiequilibrium evolution is still satisfied. The boundary conditions for the remanent MHD variables are the same as in phases II and III.

Finally, during all four phases of the simulation, open boundary conditions are applied along the lateral planes.

\subsection{Relaxation Runs}

As a consequence of the twisting motions applied during the previous phase, magnetic pressure has been injected into the system, eventually resulting in a slight inflation of the arcade. Moreover, plasma flows along the magnetic field are generated as a response of the plasma to the driving mechanism. The existence of such flows has also been discussed in Aulanier et al. (2005, 2012) and Selwa et al. (2012). In order to investigate whether or not the system has already reached the point at which it will erupt, we performed a relaxation run. In particular, at the lower boundary, we applied reflective boundary conditions to the tangential components of the velocity and we fixed the tangential component of the magnetic field to the value it has at time $t=230 t_{A}$, while the vertical component is determined by the $\nabla \cdot \mathbf{B}=0$ condition. We found that the system stores in the magnetic field almost all the current that was built up during the twisting phase as well as the shear along the polarity inversion line. As already mentioned, the system undergoes a slow expansion due to both the residual Lorentz force and the plasma flows, that, higher up in the atmosphere where $\beta \sim 1$, can efficiently perturb the magnetic field. However, this expansion is far slower when compared to the eruption phase. Finally, it is worth mentioning that since our model also takes gravity into account, we could not reset the velocities in all the numerical domain as was done, for example, by Aulanier et al. (2005) 
and Török \& Kliem (2003). Therefore, in this study, we could not disentangle the mutual contribution that the imperfect forcefree condition, i.e., the residual Lorentz force, and the plasma motions have in the field expansion.

\section{RESULTS}

\subsection{Twisting Phase}

As discussed in the previous section, in order to build up currents in the system, we apply localized twisting motions. Figure 4 shows the status of the system after such motions have been applied for $230 t_{A}$. During this driving phase, the twisting velocity never exceeds $5 \%$ of the local Alfvén speed. Therefore, the magnetic field uniformly responds to the photospheric motions and, as a consequence, magnetic shear is introduced between the negative polarity and the following positive one. As shown in the figure, a significant amount of current is also built up in the system, reaching a maximum value of about 2.7 (dimensionless code units). Due to the applied shearing motions, the magnetic field evolves from a potential state to a force-free field configuration.

The field lines close to the polarity inversion lines are also now significantly sheared higher up in the solar atmosphere. The applied twisting motions involve the full polarity, and therefore the external part of the positive following polarity is also subject to twisting motions. As a result, a J-shaped bundle of field lines, closely resembling the loop system observed in the EUV images of NOAA 9415 (Figure 1(d)), is formed. However, we stress that during this phase the photospheric motions that we apply are not intended to mimic the observed ones; instead, we aim to inject currents into the system in order to build up enough magnetic free energy to drive the eruption and to do it in such a way that the associated chirality is consistent with the observations. The chirality of the AR (sinistral or dextral) is also closely associated with the shape (forward or inverse) of the sigmoid. Figures 4(a) and (b) show the top and side views of some selected field lines color coded with the current density. The higher currents are injected near the maximum of the magnetic field and around the polarity inversion line. Moreover, Figure 4(c) also shows an isocontour of the current density. In order to better investigate the formation of the sigmoid, we integrated the vertical component of the current density in a similar fashion as discussed in Amari et al. (2000, 2011) and Aulanier et al. (2010). Figure 5 shows the integrated current density after 140 (a) and 230 (b) $t_{A}$, together with a soft X-ray telescope (SXT) image of the AR taken on 2001 April 8 at 22:47 UT (c). At first, the increase in the current distribution is observed around the two main magnetic polarities and around the polarity inversion line. In fact, this is the region which first experiences the highest shear. However, when the twist continues, the field lines that connect the outermost edges of the magnetic polarity are also affected, eventually resulting in the forward-S structure that is visible in Figure 5(b). A comparison between Figures 5(b) and (c) shows that a good qualitative agreement is found even though not all features are reproduced. The chirality of the sigmoid is reproduced as well as its fragmented structure. However, the eastern branch of the J-shaped emission is much more aligned to the north-south direction in the SXT image than in the simulation. Moreover, in the SXT image, the J-shaped loop bundle emits more than what can be determined from our images. Nevertheless, as previously discussed, our simulation closely reproduces these bundles (Figure 4(a)). The difference may be due to several reasons. The AR had a quite intense flare activity that might have

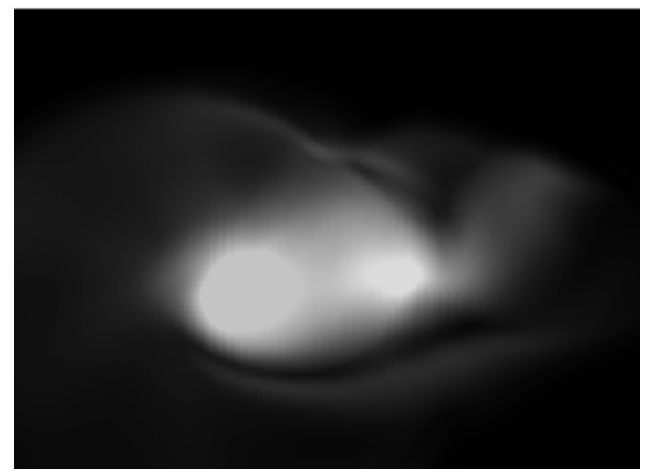

(a)

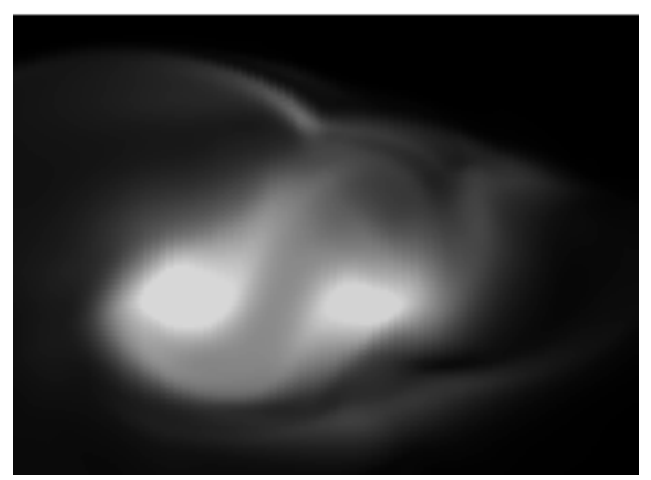

(b)

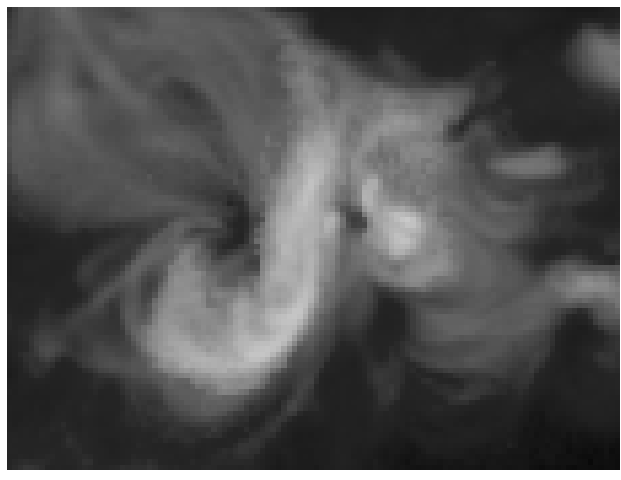

(c)

Figure 5. Integrated current density $\left(j_{z}\right)$ for the system after the twisting motions have been applied for (a) $140 t_{A}$ and (b) $230 t_{A}$. (c) SXT image of the AR taken on 2001 April 8 at 22:47 UT.

resulted in a higher emission with respect to the one associated with the ohmic heating, or more twist could be present with respect to our simulation. In fact, if we continue twisting up to $350 t_{A}$, a significant increase of the integrated current density in the region corresponding to the $\mathrm{J}$-bundle is observed. However, at this point, the field lines that close higher up in the atmosphere are significantly twisted and the system approaches a disruption phase similar to what was found in Amari et al. (1996). This is beyond the scope of this paper, where we want to investigate whether or not the shearing motions that characterized the east-most positive polarity might have been able to eventually destabilize the system, resulting in an eruption.

\subsection{Erupting Phase}

The initial state of this phase is the configuration of the system after the twisting motions have been applied for $230 t_{A}$. As discussed in the previous section, this configuration can be 


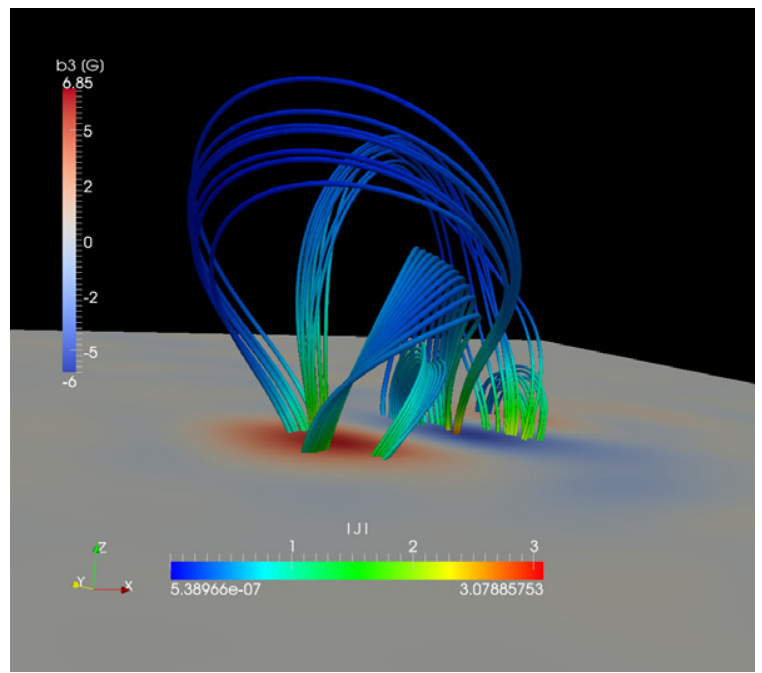

(a)

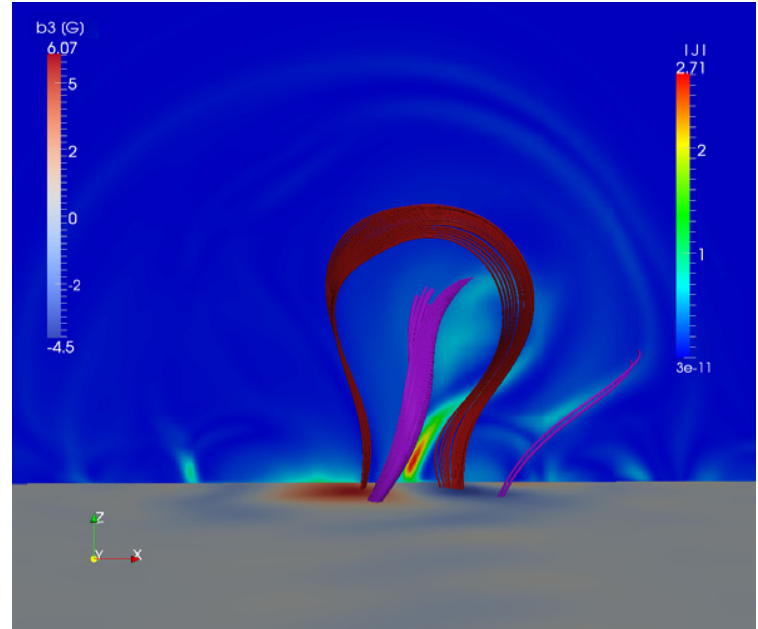

(c)

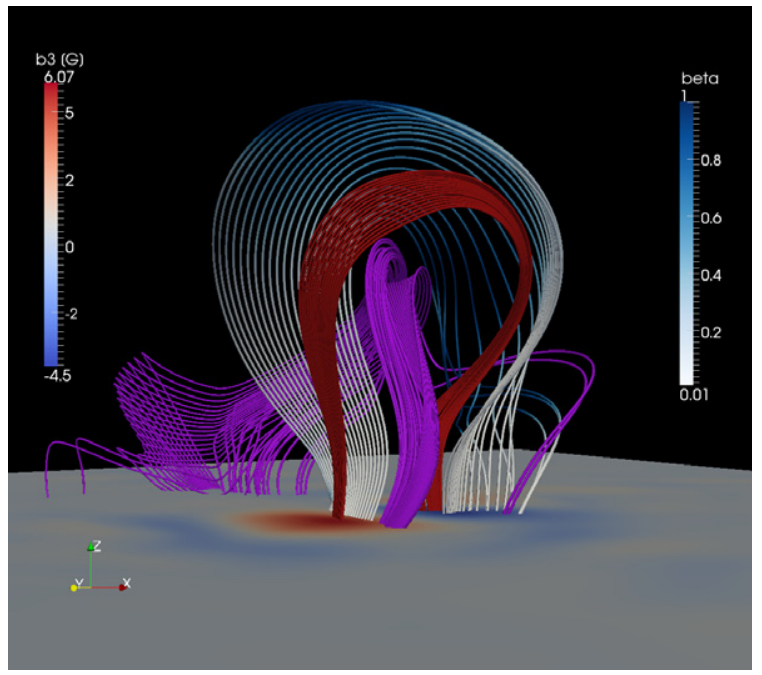

(b)

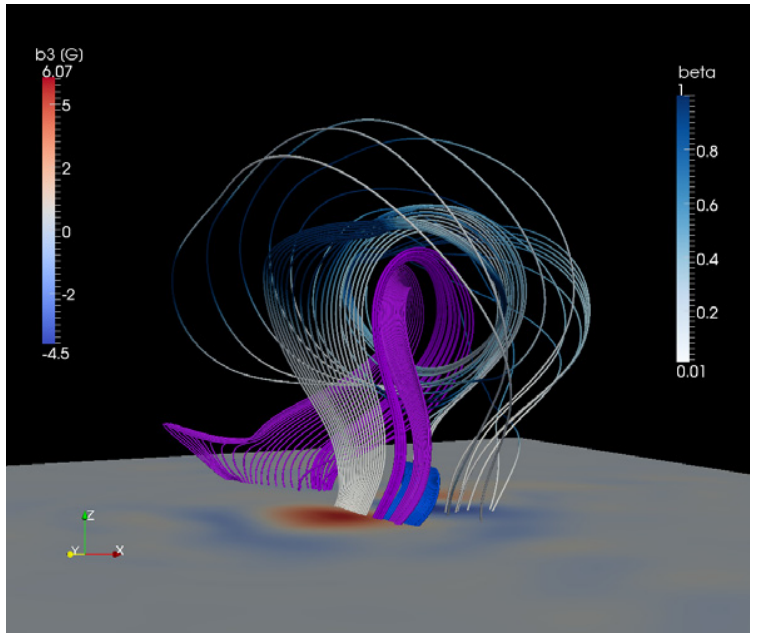

(d)

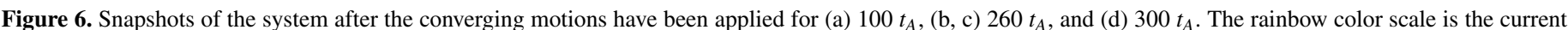

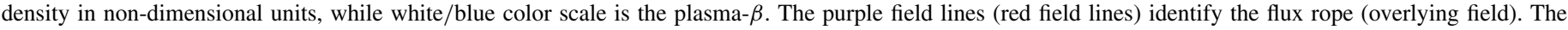
bright blue field lines in panel (d) highlight selected post-flare loops.

(A color version of this figure is available in the online journal.)

considered stable within the timescales that we are interested in. We therefore reset the time to zero and apply the shearing motions described in Section 3.2.

Figure 6(a) shows the configuration of the system after the converging motions have been applied for about $100 t_{A}$. Selected field lines are color coded with the current density. As a consequence of the applied convergence motions, the initial equilibrium is perturbed and the highly sheared field lines around the polarity inversion line start to rise.

Figures 6(b) and (c) show the configuration of the system $260 t_{A}$ after the convergence shearing motions began. Due to the convergence motions, positive magnetic flux is transported toward the polarity inversion line and magnetic reconnection sets in between the highly sheared field lines, eventually resulting in the formation of a current-carrying flux rope (purple field lines). However, due to the reconnection and to the threedimensional nature of the problem, the newly formed flux rope is not a closed plasmoid as discussed in the axisymmetric simulations (Zuccarello et al. 2008, 2009; Soenen et al. 2009), but presents a strongly twisted magnetic field with both ends anchored in the lower boundary. Evidence of the reconnection is visible in Figure 6(c) where the current density distribution along the $x z$-plane is shown together with part of the flux rope and some selected overlying field lines. A current density increase is observed below the flux rope. This is evidence for the formation of such a reconnection layer. We also analyzed the plasma flows around that region and we found that flows toward the reconnection region are observed along the $x$-direction, while strong upward and downward plasma outflows, reaching velocities of about $1400 \mathrm{~km} \mathrm{~s}^{-1}$, are observed in the vertical direction. These pieces of evidence, in combination with the observed increase in the thermal energy (Figure 9), strongly support the formation of a reconnection layer.

Figure 6(b) also shows another bundle of field lines color coded with the value of the plasma- $\beta$. These field lines still belong to the overlying magnetic field, but are more twisted than the red field lines. During the upward propagation of the flux rope and due to the reconnection below it, more and more overlying magnetic field is converted into magnetic field of the flux rope as shown in Figure 6(d). During this phase, the 


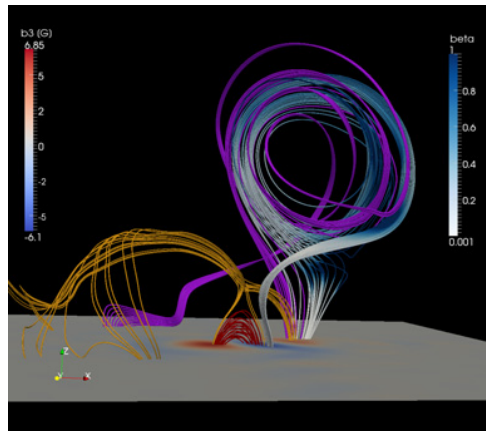

(a)

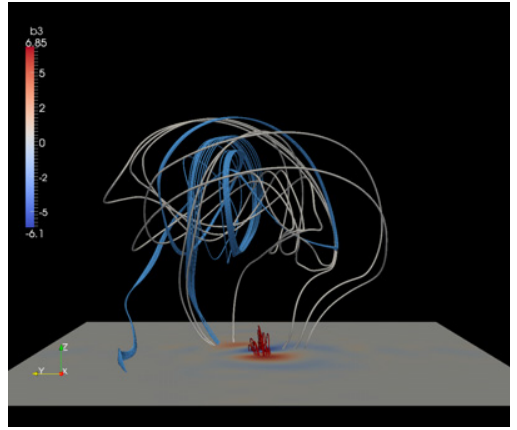

(b)

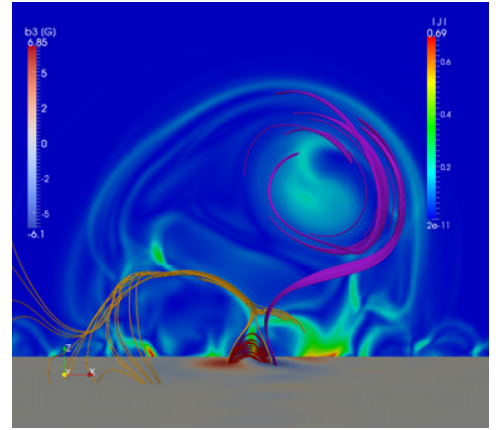

(c)

Figure 7. Different views of the system after $700 t_{A}$. See the text for an explanation of different color field lines.

(A color version of this figure is available in the online journal.)

current J-shaped features continue to approach and eventually will merge after about $340 t_{A}$, when a significant amount of the overlying field has been transferred to the flux rope.

As evident from Figure 6(b), the flux rope presents a quite fragmented structure. In fact, while one magnetic foot point is well located in the positive polarity, being reminiscent of the highly sheared field lines, the other foot point is quite diffuse. This may be a consequence of the plasma flows generated as a response of the system to the applied boundary motions. These supersonic flows around the flux rope generate a shock that compresses the magnetic field generating a current layer where a change in the magnetic field topology can occur.

After $t=450 t_{A}$, we linearly switch off the convergence motions and let the system evolve. Due to the higher magnetic pressure, the flux rope continues to move upward, eventually interacting with the overlying magnetic field. Figure 7 shows the configuration of the system after $700 t_{A}$. At this stage of the simulation, the null point separates the flux rope field from the post-flare loops that after the reconnection are now approaching a more potential state (Figure 7(a)). Figure 7(b) shows a side view of the system. Due to the asymmetry of the configuration and to the perturbation of the plasma, the flux rope presents a quite fragmented structure. From the positive polarity of the $\delta$-spot, field lines enter the flux rope, make some turns, and leave it in agreement with Démoulin et al. (1996). In particular, light grey field lines are connected with the southern part of the negative polarity, while the light blue ones are connected with a negative polarity at the north of the positive magnetic field spot. Figure 7(c) shows a vertical cut of the current density distribution. Within the flux rope, an increase in the current density is observed, as expected as a consequence of the highly twisted field lines. Moreover, in front of the flux rope, a mushroom-shaped current layer is observed. This is spatially correlated with the shock and with the transition between a medium where the plasma- $\beta$ is smaller than one to a medium where it is larger than one.

Figure 8 shows the $\mathrm{CME}$ while it is propagating into the numerical domain after $1000 t_{A}$. A compressed leading edge, associated with the shock front, is observed. Moreover, an increase in the density is also observed within the flux rope followed by a density depletion. The figure also shows selected field lines (red) that connect the two main polarities. As is evident, the magnetic field that reconnects behind the flux rope has a quite potential state.

Finally, as already mentioned, during its expansion, the flux rope propagates in a medium where the plasma- $\beta$ is originally higher than one. However, due to the density depletion from one side and the higher magnetic field carried by the flux rope
(Figure 8(b)) with respect to the original background field on the other side, the plasma- $\beta$ is always smaller than one within the CME volume with a sharp change along the shock that actually interfaces the unperturbed background medium with the magnetized plasma carried away with the flux rope. Such shocks at the front of the CME have often been observed (Vourlidas \& Ontiveros 2009) and have been simulated by Roussev et al. (2008).

\subsection{CME Energy and Velocity}

For the present numerical simulation, we solved the full set of MHD equations, including the energy equation. This enables us to consistently investigate not only the evolution of the magnetic energy, but also the evolution of the kinetic and internal energy. Figure 9 shows the evolution of the magnetic, kinetic, and internal energy relative to the initial magnetohydrostatic equilibrium as a function of time. As a consequence of the applied twisting motions, the magnetic energy increases almost linearly up to about $t=230 t_{A}$. After that, during a transition phase where we continuously switch from twisting to convergence motions, the magnetic energy starts to decrease. After about $400 t_{A}$, this decrease becomes even steeper and eventually reaches the initial value. Toward the end of the simulation, the magnetic energy seems to increase, and this may be a consequence of the influence of the boundary motions on the initial magnetic field distribution at the inner boundary. In fact, although during the twisting phase the motions have been applied along isocontours of $B_{z}$, therefore leaving the flux distribution of $B_{z}$ unmodified, during the convergence phase, in response to such motions, the distribution of $B_{z}$ at the inner boundary changed slightly and eventually resulted in a post eruption magnetic field with slightly higher energy than the initial one. However, at this stage, the CME front reached the outer boundaries and therefore a detailed analysis cannot be performed.

During the twisting phase, an increase in the internal and kinetic energy is observed. This is due to the plasma motions generated in response to the magnetic field evolution. However, when the decrease in the magnetic energy becomes steeper, the increases in the kinetic and internal energy also become more evident. Finally, when the shock reaches the boundaries of the simulation domain, the internal and the kinetic energies start to decrease.

In order to derive the CME height-time plots, we considered a vertical cut along the $x z$-plane and identified the core of the CME looking at the position of the maximum of $B_{y}$ and at the zero of $B_{x}$. We also located the front of the CME by following on 


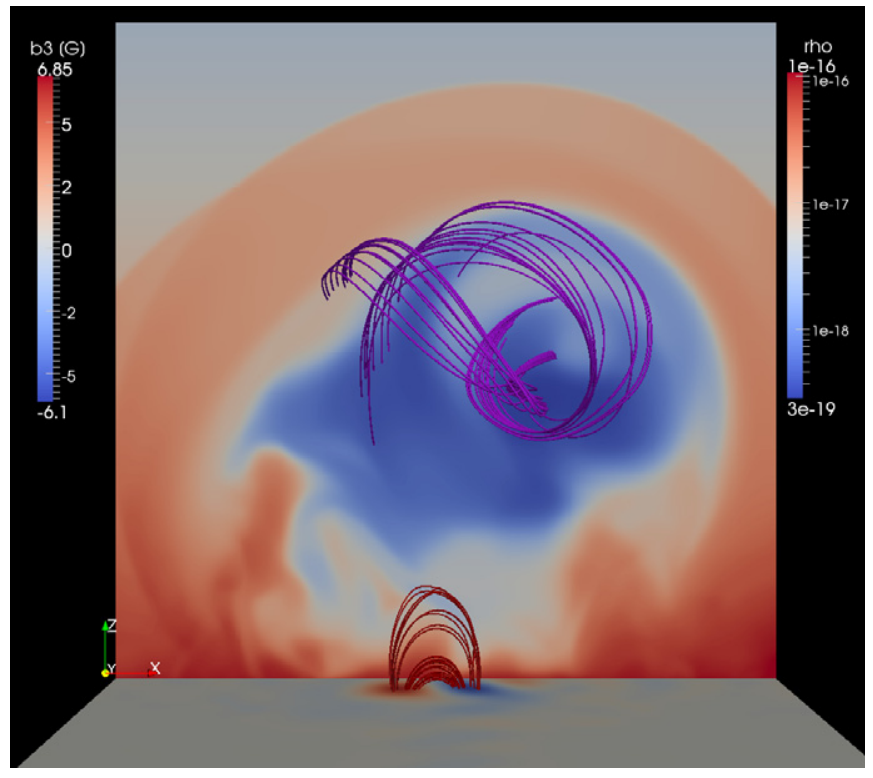

(a)

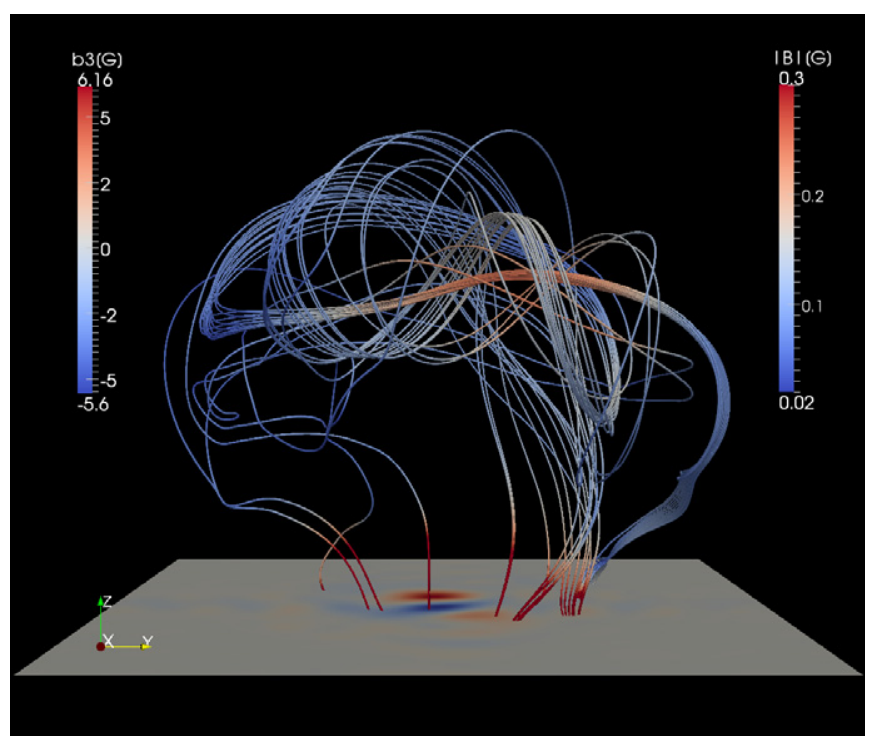

(b)

Figure 8. Views of the system after $1000 t_{A}$. (a) Front view: a vertical cut of the density is shown together with the flux rope field (purple field lines) and the post-flare loops (red field lines). (b) Side view: field lines are color coded with the magnetic field intensity.

(A color version of this figure is available in the online journal.)

the same plane the shock in front of the CME. Figure 10(a) shows such height-time plots. The front and the core of the CME seem to move at similar speeds during the initial phase of the eruption; however, while the CME is propagating within the numerical domain, the core seems to slow down. This can be deduced from Figure 10(b) where the speed has been calculated. After a faster phase, both the core and the front of the CME slow down to a speed of about $400 \mathrm{~km} \mathrm{~s}^{-1}$ and afterward the CME front seems to converge toward a speed of about $550 \mathrm{~km} \mathrm{~s}^{-1}$, while the core approaches a speed of about $250 \mathrm{~km} \mathrm{~s}^{-1}$. However, this is only a lower limit for the CME core velocity. In fact, with this method, we only follow the vertical motion of the core, but the CME also undergoes a deflection in the $x y$-plane. In order to further constrain the CME core speed, we looked at the plasma velocity in a plane that contains the flux rope axis, i.e., a plane parallel to

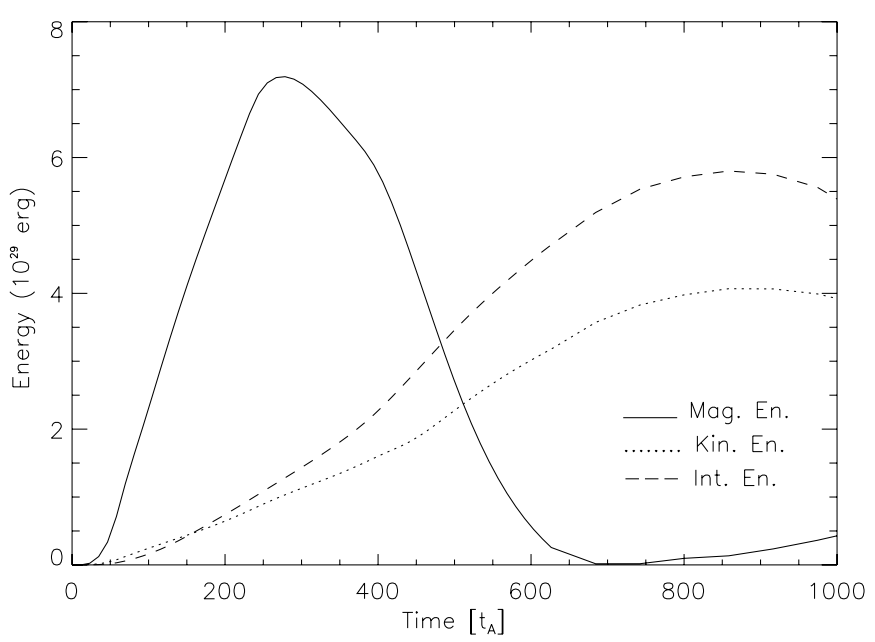

Figure 9. Time evolution of the magnetic (solid line), kinetic (dotted line), and internal (dashed line) energy relative to the initial magnetohydrostatic equilibrium.

the $y z$-plane and we found plasma flows of about $430 \mathrm{~km} \mathrm{~s}^{-1}$. This is consistent with the fact that the distance between the CME front and core does not vary too much in our simulations.

\section{DISCUSSION}

The aim of the present paper was to present a data-inspired model for the initiation and early evolution of CMEs. As an example of the possible applications of the model, the evolution of AR NOAA 9415 was simulated. Using data-inspired initial and boundary conditions, we succeeded to initiate a CME in this AR. In particular, among the several CMEs that occurred in AR 9415, the filament eruption on April 10 was implicitly selected to be the target of our simulation. The CME associated with the filament eruption was a really fast halo CME $\left(\sim 2000 \mathrm{~km} \mathrm{~s}^{-1}\right)$. The simulation did not succeed to reproduce the desired speed, but resulted in a more modest event $\left(\sim 550 \mathrm{~km} \mathrm{~s}^{-1}\right)$. This can be due to several reasons. First, the CME was associated with a filament, and we believe that convergence motions resulted in the destabilization of this filament, while in the simulation, the convergence motions formed the flux rope that immediately underwent the eruption. As a result, at the moment of the eruption, the flux rope might not have had enough poloidal flux to drive a faster eruption. A new simulation in which a flux rope is inserted in the pre-eruption configuration might help to clarify this point. Second, in the simulation, the configuration of the overlying field was only the result of a potential field extrapolation, and therefore possible connections with other ARs and with the global solar magnetic field are not considered in our model (Jacobs et al. 2009; Jacobs \& Poedts 2012). As discussed in Amari et al. (2007), the ability of flux cancellation to initiate CMEs is independent from the topology of the overlying field, but a multipolar configuration might lead to faster eruptions. Inserting the present model into a more global simulation might give interesting insights on the early evolution of the CME. Finally, the finite, larger than one plasma- $\beta$ might have played a contribution. In fact, due to the simplified solar atmosphere selected for this simulation, after about $1.6 R_{\odot}$ the plasma- $\beta$ becomes too high. As a result, the inertia of the plasma slows down the eruption. The inclusion of the solar wind, that becomes relevant starting from that height, will result in a more realistic plasma- $\beta$ profile and probably in a faster eruption.

The low corona is a low plasma- $\beta$ environment and many simulations have been preformed assuming $\beta=0$ (Amari 


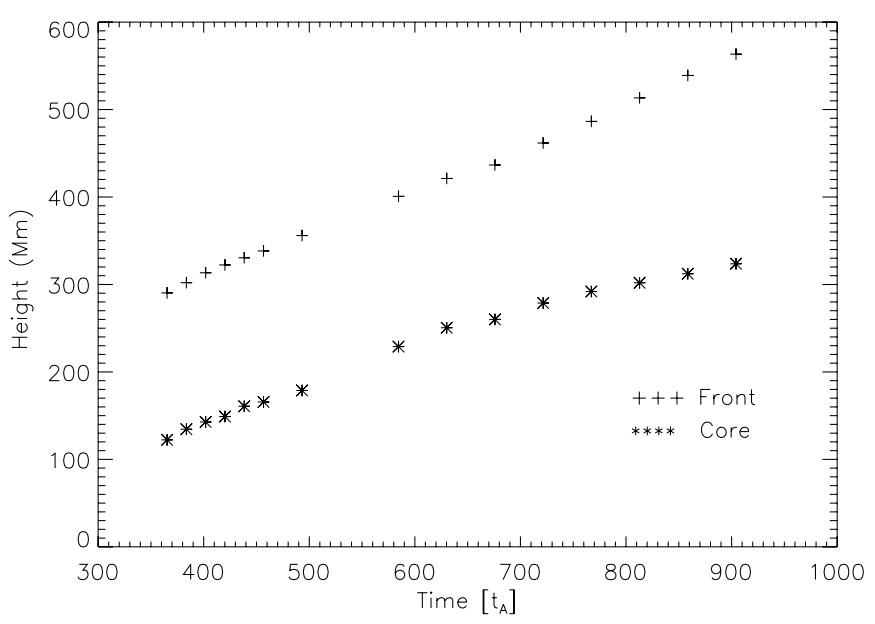

(a)

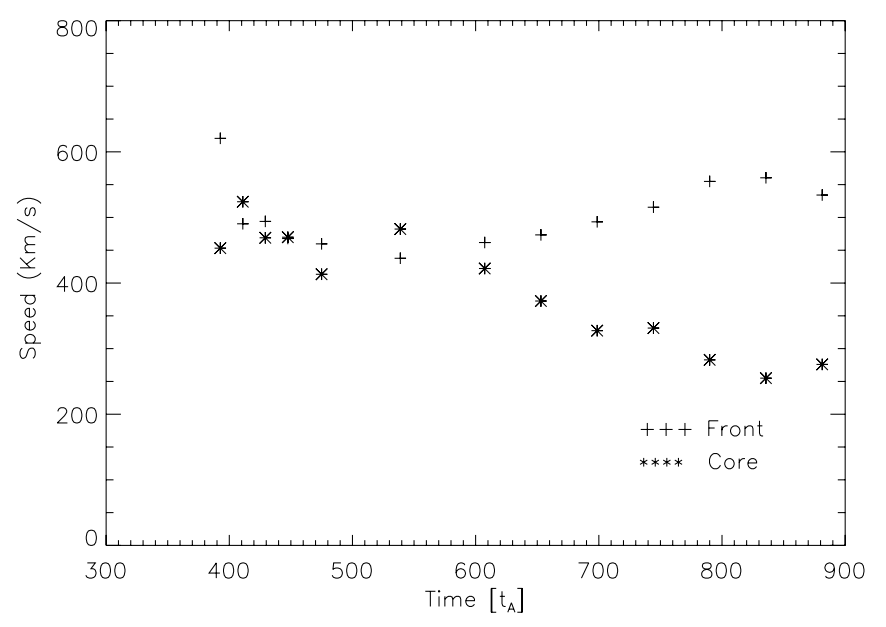

(b)

Figure 10. Height-time (a) and velocity-time (b) plots for the center of the flux rope (asterisk) and CME front (pluses).

et al. 2003a, 2011; Aulanier et al. 2010). However, our simulation shows that considering a finite- $\beta$ plasma can affect at least the propagation of the CME, if not its initiation. These results support the study of Fuentes-Fernández et al. (2010). Furthermore, as also suggested by Aulanier et al. (2012), the inclusion of a finite- $\beta$ plasma allows a better understanding of the reconnection-related magnetic energy decrease. In fact, the magnetic energy can be converted into particle acceleration or heat. Our simulation shows a significant increase in the internal energy during the flare reconnection (Figure 9). This increase is even more pronounced than the one in the kinetic energy, eventually supporting the considerations of Aulanier et al. (2012) on the relevance of heating processes during flares. Similar to Aulanier et al. (2010, 2012), the post-flare loops are also observed in our simulation (Figure 8(a)). The transition from the initially high sheared configuration to a more potential one proceeds in a gradual fashion, starting from the loops closer to the inner boundary to loops that extend higher up in the solar atmosphere.

The overall evolution of our simulation, including the flux rope formation, is similar to the asymmetric case described in Aulanier et al. (2010). The authors concluded that the tethercutting reconnection contributed to the formation of the flux rope and to bringing its axis to a height where the onset criterion for the torus instability was satisfied, eventually resulting in the full eruption of the flux rope. Their energy plot shows that the kinetic energy increases some Alfvén times before the decrease in the magnetic energy began. This is compatible with the scenario in which the flux rope starts propagating first due to the torus instability, and then the flare reconnection sets in, further contributing to the energy release. In our case, the change of the slope in the kinetic energy is synchronized with the change in the slope for the magnetic energy. We therefore believe that in our case flux cancellation played a primary role in triggering and driving the CME. This was the case in Amari et al. (2003a), where the flux rope is never in equilibrium. However, to conclusively address this issue, a more detailed equilibrium analysis is needed. This will be the focus of a next paper.

During the eruption, the filament underwent a southwest deflection and the TRACE evolution also shows its interaction with the northern filament. The simulated flux rope also undergoes a similar deflection during its propagation. As we discussed in the previous section, the topology of the eruption is quite complex and the $\delta$-spot plays a significant role in determining the connectivity of the flux rope. One foot point of the flux rope is anchored in the $\delta$-spot during the whole propagation phase. Starting from this foot point, it is possible to identify two ensembles of field lines: (1) one connecting the positive polarity of the $\delta$-spot with the negative intrusion at the south of the following polarity and (2) one connecting the $\delta$-spot with a negative polarity at the north of it, where the second major filament was located. This magnetic configuration may explain both the deflection and the perturbation of the second filament. The deflection can be a consequence of a pressure imbalance between the northwest foot point (the one anchored in the $\delta$-spot) and the southern one (Zuccarello et al. 2012; Bemporad et al. 2012). The interaction with the second filament is the consequence of the change in the magnetic field topology.

During the CME propagation, a strong density compression is observed. Such a compression has also been reported in more global simulations (Cohen et al. 2009; Downs et al. 2011; Jacobs $\&$ Poedts 2012), and has been interpreted as the compression front caused by the interaction between the expanding magnetic field and the overlying field (Jacobs \& Poedts 2012). Density enhancements have also been reported by Selwa et al. (2012) and have been interpreted as propagating EIT waves. In our simulation, this density enhancement is the result of the shock generated during the propagation of the $\mathrm{CME}$ and does not seem to be related to the interaction between the flux rope and the overlying field. It is more likely the result of the interaction between the faster plasma associated with the CME and the ambient one. However, global MHD simulations are needed in order to better understand the plasma-plasma and plasma-magnetic-field interactions.

In our simulation, a new class of asymmetric convergence motions has been considered. The existence of such asymmetric convergence motions has been reported, for example, by Green et al. (2011). The authors concluded that these motions may have resulted in the formation and consequent eruption of a flux rope. We have shown that such convergence motions can initiate an eruption when a force-free-magnetic field configuration is considered. These results further contribute to the analysis on the flux cancellation mechanism conducted by Amari and coauthors. Amari et al. (2011) have shown that in order to form a flux rope from a force-free configuration, it is enough that only a part of the magnetic polarities is advected toward the polarity inversion line. The applied convergence motions were symmetric with respect to this polarity inversion line. 
Our results also confirm their conclusion when asymmetric convergence motions are considered. Furthermore, Amari et al. $(2000,2007,2010,2011)$ found that when the disruption occurs, an amount between the $4.5 \%$ and $30 \%$ of the initial flux is canceled at the polarity inversion line. At the moment when the flare reconnection sets in, we find that the unsigned flux around the polarity inversion line decreases by about $4 \%$. Therefore, our simulation supports the conclusions of Amari et al. (2010): a small amount of flux cancellation is sufficient to trigger a large disruption. Finally, our results are also aligned with the community effort to perform numerical simulations where the applied boundary conditions resemble the observations. In fact, we have argued that this new class of asymmetric converging motions was observed for at least three ARs, specifically the one discussed in this paper and the ones presented in Green et al. (2011) and Cheng et al. (2010).

\section{SUMMARY}

We performed a full three-dimensional, ideal MHD simulation of the AR NOAA 9415 in a non-zero plasma- $\beta$ regime. Starting from a potential field extrapolation, we applied localized vortex motions in order to build up magnetic free energy into the system. The obtained magnetic field configuration had the same chirality as the considered AR.

Due to the applied convergence motions that were inspired by the observed ones, we were able to initiate a CME. The flux rope is formed and quickly propagates outward. The overall morphology of the flux rope is similar to the simulation of Aulanier et al. (2010), where an asymmetric dipole was considered. However, in our case, we do not only have an asymmetric dipole, but we also have a $\delta$-spot. The $\delta$-spot actually plays a significant role during the propagation of the CME. The flux rope presents a quite fragmented structure with one of the two foot points anchored on the positive polarity of the $\delta$-spot, eventually facilitating the deflection of the CME during its propagation.

Even though the flux rope foot points are anchored in the photosphere, part of the plasma is confined inside the flux rope and carried away with it against the solar gravity. The system therefore presents a three-part structure consisting of a compressed front, an "empty" cavity, and a high density core. The compressed leading edge has a higher density with respect to the CME core.

The CME propagates in a medium where the plasma- $\beta$ is higher than one; however, during its propagation, the $\mathrm{CME}$ perturbs the surrounding medium and due to the higher, with respect to the background, magnetic field carried within the flux rope and to the density depletion, within the CME volume the plasma- $\beta \approx 0.1$. This transition of the plasma- $\beta$ from higher to lower than one is often observed within magnetic clouds. However, in our simulation, the solar wind is not considered and therefore these considerations should be taken carefully. The extension of the present model including the solar wind in a spherical geometry will be the subject of a future paper.

F.P.Z. thanks G. Aulanier, P. Demoulin, and C. Jacobs for the intriguing and stimulating discussions. These results were obtained in the framework of the projects GOA/2009-009 (KU Leuven), G.0729.11 (FWO-Vlaanderen), and C 90347 (ESA Prodex 9). The research leading to these results has also received funding from the European Commission's Seventh Framework Programme (FP7/2007-2013) under the grant agreements SOLSPANET (project No. 269299, www.solspanet.eu/solspanet), SPACECAST (project No.
262468, fp7-spacecast.eu), eHeroes (project No. 284461, http://soteria-space.eu/eheroes/html), and SWIFF (project No. 263340, www.swiff.eu). For the computations, we used the infrastructure of the VSC "Flemish Supercomputer Center, funded by the Hercules foundation and the Flemish Government" department EWI and the JADE-CINES cluster (Montpellier-France) within the Europa-HPC2 project and the DARI project x2012046842.

\section{REFERENCES}

Amari, T., Aly, J.-J., Luciani, J.-F., Mikic, Z., \& Linker, J. 2011, ApJ, 742, L27 Amari, T., Aly, J. J., Mikic, Z., \& Linker, J. 2007, ApJ, 671, L189

Amari, T., Aly, J.-J., Mikic, Z., \& Linker, J. 2010, ApJ, 717, L26

Amari, T., Luciani, J. F., Aly, J. J., Mikic, Z., \& Linker, J. 2003a, ApJ, 585, 1073

Amari, T., Luciani, J. F., Aly, J. J., Mikic, Z., \& Linker, J. 2003b, ApJ, 595,1231

Amari, T., Luciani, J. F., Aly, J. J., \& Tagger, M. 1996, ApJ, 466, L39

Amari, T., Luciani, J. F., Mikić, Z., \& Linker, J. A. 2000, ApJ, 529, L49

Asai, A., Shibata, K., Ishii, T. T., et al. 2009, J. Geophys. Res. (Space Phys.), 114,0

Aulanier, G., Démoulin, P., \& Grappin, R. 2005, A\&A, 430, 1067

Aulanier, G., Janvier, M., \& Schmieder, B. 2012, A\&A, 543, A110

Aulanier, G., Török, T., Démoulin, P., \& DeLuca, E. E. 2010, ApJ, 708, 314

Bemporad, A., Zuccarello, F. P., Jacobs, C., Mierla, M., \& Poedts, S. 2012, The Sun 360, Sol. Phys., 153

Cheng, X., Ding, M. D., \& Zhang, J. 2010, ApJ, 712, 1302

Cohen, O., Attrill, G. D. R., Manchester, W. B., IV., \& Wills-Davey, M. J. 2009, ApJ, 705, 587

Colella, P., \& Woodward, P. R. 1984, J. Comput. Phys., 54, 174

Cristiani, G., Martinez, G., Mandrini, C. H., et al. 2007, Sol. Phys., 240, 271

Dedner, A., Kemm, F., Kröner, D., et al. 2002, J. Comput. Phys., 175, 645

Démoulin, P., Priest, E. R., \& Lonie, D. P. 1996, J. Geophys. Res., 101, 7631

Downs, C., Roussev, I. I., van der Holst, B., et al. 2011, ApJ, 728, 2

Forbes, T. G., \& Isenberg, P. A. 1991, ApJ, 373, 294

Forbes, T. G., \& Priest, E. R. 1995, ApJ, 446, 377

Fuentes-Fernández, J., Parnell, C. E., \& Hood, A. W. 2010, A\&A, 514, A90

Gary, G. A. 2001, Sol. Phys., 203, 71

Gopalswamy, N., \& Yashiro, S. 2011, ApJ, 736, L17

Green, L. M., Kliem, B., \& Wallace, A. J. 2011, A\&A, 526, A2

Harten, A., Lax, P. D., \& Leer, B. V. 1983, SIAM Rev., 25, 35

Jacobs, C., \& Poedts, S. 2012, Advances in European Solar Physics, Sol. Phys., 25

Jacobs, C., Roussev, I. I., Lugaz, N., \& Poedts, S. 2009, ApJ, 695, L171

Keppens, R., Meliani, Z., van Marle, A. J., et al. 2012, J. Comput. Phys., 231, 718

Lin, H., Kuhn, J. R., \& Coulter, R. 2004, ApJ, 613, L177

Lionello, R., Linker, J. A., \& Mikić, Z. 2009, ApJ, 690, 902

Mancuso, S. 2011, Sol. Phys., 273, 511

Otto, A., Büchner, J., \& Nikutowski, B. 2007, A\&A, 468, 313

Powell, K. G., Roe, P. L., Linde, T. J., Gombosi, T. I., \& de Zeeuw, D. L. 1999, J. Comput. Phys., 154, 284

Roussev, I. I., Lugaz, N., \& Sokolov, I. V. 2008, in AIP Conf. Ser. 1039, Particle Acceleration and Transport in the Heliosphere and Beyond: 7th Annual International Astrophysics Conference, ed. G. Li, Q. Hu, O. Verkhoglyadova, G. P. Zank, R. P. Lin, \& J. Luhmann (Melville, NY: AIP), 286

Selwa, M., Poedts, S., \& DeVore, C. R. 2012, ApJ, 747, L21

Soenen, A., Zuccarello, F. P., Jacobs, C., et al. 2009, A\&A, 501, 1123

Sterling, A. C., Chifor, C., Mason, H. E., Moore, R. L., \& Young, P. R. 2010, A\&A, 521, A49

Su, Y., Surges, V., van Ballegooijen, A., DeLuca, E., \& Golub, L. 2011, ApJ, 734,53

Tanaka, T. 1994, J. Comput. Phys., 111, 381

Török, T., \& Kliem, B. 2003, A\&A, 406, 1043

van Ballegooijen, A. A., \& Martens, P. C. H. 1989, ApJ, 343, 971

Vourlidas, A., \& Ontiveros, V. 2009, in AIP Conf. Ser. 1183, Shock Waves in Space and Astrophysical Environments: 18th Annual International Astrophysics Conference, ed. X. Ao \& G. Z. R. Burrows (Melville, NY: AIP), 139

Welsch, B. T., Christe, S., \& McTiernan, J. M. 2011, Sol. Phys., 274, 131

Zhang, J., Li, L., \& Song, Q. 2007, ApJ, 662, L35

Zuccarello, F. P., Bemporad, A., Jacobs, C., et al. 2012, ApJ, 744, 66

Zuccarello, F. P., Jacobs, C., Soenen, A., et al. 2009, A\&A, 507, 441

Zuccarello, F. P., Soenen, A., Poedts, S., Zuccarello, F., \& Jacobs, C. 2008, ApJ, 689, L157 